\title{
ESTUDIO DE FACTIBILIDAD PARA PUBLICITAR PRODUCTOS EMPRESARIALES CON CÓDIGOS QR
}

\section{FEASIBILITY STUDY TO ADVERTISE BUSINESS PRODUCTS WITH QR CODES}

Fernando Silva Ordoñez ${ }^{1}$ Patricio Medina Chicaiza ${ }^{2}$ Soledad Sarzosa Rivera ${ }^{3}$

1. Ingeniero de Empresas. Magister en Gestión Estratégica Empresarial. Magister en Administración y Marketing. Universidad Técnica de Ambato, Docente de la Facultad de Ciencias Administrativas, Ambato - Ecuador. E-mail: ivanfsilva@uta.edu.ec

2. Ingeniero de Sistemas y Computación. Magister en Tecnologías de la Información. Pontificia Universidad Católica del Ecuador, Docente de la Escuela de Ingeniería en Sistemas. Universidad Técnica de Ambato, Docente de la Facultad de Ciencias Administrativas, Ambato - Ecuador. E-mail: pmedina@pucesa.edu.ec; ricardopmedina@uta.edu.ec

3. Estudiante Universitaria. Universidad Técnica de Ambato. Ambato - Ecuador.

E-mail: estefaniasarzosa@gmail.com

Citación sugerida:

Silva Ordoñez, F., Medina Chicaiza, P., y Sarzosa Rivera, S. (2016). Estudio de factibilidad para publicitar productos empresariales con códigos QR. 3C Tecnología: glosas de innovación aplicadas a la pyme, 5(3), 1-19. DOI: <http://dx.doi.org/10.17993/3ctecno.2016.v5n3e19.1-19/>. 


\section{RESUMEN}

El presente trabajo tiene como objetivo realizar un estudio de factibilidad para publicitar productos empresariales con códigos QR en la ciudad Ambato, en Ecuador. Se ejecutó un diagnóstico a través del sistema de marco lógico, posteriormente se realizó un estudio de mercado, técnico-administrativo y económico-financiero con el propósito de determinar la factibilidad y viabilidad de esta idea de negocio a través de la evaluación económica y financiera. Además, con el marco lógico se definieron los problemas e involucrados directos e indirectos. Mediante el análisis de mercado se determinó una demanda potencial insatisfecha, debida a la insuficiente innovación en los productos publicitarios por parte de las empresas. En el estudio técnico-administrativo se destaca el marco legal de la empresa y la ingeniería del proyecto, estableciendo los insumos, servicios básicos, equipos, herramientas y personal de contacto indispensable, además de la localización, tamaño óptimo y los procesos de servicio. En la fase económica-financiera se centró en hallar la inversión inicial, determinar los ingresos brutos y gastos, reflejados en el estado de resultados y el balance general. Finalmente, la evaluación económica y financiera mostró la factibilidad y viabilidad del proyecto mediante los evaluadores en tiempo presente y futuro. Dentro de las conclusiones se evidenciaron resultados positivos para el valor actual neto, tasa interna de retorno y la relación costo/beneficio, por lo que la creación de una empresa que publicite productos empresariales con códigos QR conseguirá un creciente segmento de mercado.

\section{ABSTRACT}

The objective of the following paper is to show the feasibility of publicizing business products in the city of Ambato, in Ecuador. A diagnosis was made through a systematic logical frame, followed by a market study, technical-administrative and financial-economical with the purpose of determining the feasibility and viability of this business idea through a financialeconomical study. Furthermore, with the logical frame the direct and indirect problems and those directly or indirectly involved were determined. By means of the market analysis a potentially unsatisfied demand, due to insufficient innovation of the products publicized by the companies was identified. In the technical-administrative study the highlighted areas were the legal frame, the engineering of the project, establishing the supplies, basic services, equipment, tools, and contact with personnel were indispensable, as well as the location, its ideal size and the viability processes. In the economic-financial phase, obtaining the initial investment, determining the gross earnings and expenses, reflected in the general balance. Finally, the economic and financial evaluation showed the feasibility and viability of the project by way of the evaluators in the present and future. In conclusion we have evidenced positive results in the net present value, internal rate of return and the relationship of cost-benefit, in which the creation of a company that publicizes business product's with QR codes will get a growing market segment. 


\section{PALABRAS CLAVE}

Estudio de factibilidad, marco Lógico, códigos QR, estudio técnico-Administrativo, estudio económico-financiero, promoción, publicidad.

\section{KEY WORDS}

Feasibility study, logical framework, QR codes, technical-administrative study, financialeconomic study, promotion, publicity. 


\section{INTRODUCCIÓN}

Es importante señalar que, en referencia al Instituto Nacional de Estadísticas y Censos (INEC, 2015), el 51.3\% de la población ecuatoriana en el año 2013 tienen por lo menos un celular activo; de las cuales el $16.9 \%$ de las personas tienen un teléfono inteligente o SMARTPHONE, frente al $8.4 \%$ del 2011. Es decir, 8.5 puntos más, lo que indica la accesibilidad a herramientas tecnológicas, aplicaciones y al mismo internet, que proporciona una interconectividad entre personas, empresas e instituciones sin importar el sitio geográfico. Con estas cifras, enfocar campañas publicitarias basadas en códigos QR permite aprovechar el incremento constante del uso de teléfonos inteligentes, logrando impacto visual y auditivo beneficioso para los intereses de la empresa.

En este trabajo, es importante considerar aspectos como: el término proyecto socioproductivo que se enfoca a impulsar el desarrollo de actividades generadoras de bienes y servicios útiles para la comunidad, generando redes productivas para satisfacer las necesidades de la sociedad, como coinciden Iñigo Carrión y Losune Berastegui (2010); Martínez Montes y Pellicer Armiñana 2011); Zilvetty Torrico (2013).

El marco lógico o sus siglas en inglés EML es una herramienta muy útil para elaborar un proyecto socio-productivo, como especifican los autores De la Torre (2010); Martínez y Pellicer (2011), que lo señalan como un instrumento que determina problemas y necesidades de un entorno, mediante una estructura de planificación para cumplir con los objetivos y llegar a resultados de una manera lógica y sistemática. A través del marco lógico, el estudio de mercado permite cuantificar la oferta y demanda, los precios y el proceso de comercialización, para comprobar la posibilidad real de penetración de un producto, bien o servicio en el mercado actual, como lo detalla Baca Urbina (2010). De esta forma se obtiene la demanda insatisfecha del segmento a analizar.

Así mismo, el aporte de Limas (2012) especifica que el estudio técnico administrativo contempla los aspectos técnicos operativos necesarios en el uso eficiente de los recursos disponibles para la producción de un bien o servicio deseado y en el cual se analizan la determinación del tamaño óptimo del lugar de producción, localización, instalaciones y organización requeridos.

Otro aspecto importante lo señala el Banco Interamericano de Desarrollo (2001), donde manifiesta que la evaluación financiera y económica pretende cuantificar y valorar el proyecto con criterios establecidos, de tal forma que la decisión de ejecutarlo, se base en indicadores confiables. De forma similar, Cubbage, Financiera y Frey (2011, p. 7) agregan que estos métodos son complejos, y el balance de este informe discute estos valores económicos y financieros y sus técnicas de medición. Razón por la cual esta etapa dentro del proceso de creación de una empresa es indispensable para determinar la factibilidad de la misma a través de indicadores presentes y futuros, como se demuestra a continuación.

Los códigos QR en pleno siglo XXI se consideran la herramienta innovadora y creativa de publicidad, marketing, posicionamiento y transmisión de información, que se pueden aplicar en cualquier tipo de empresas. De acuerdo a Beltrán López (2014) son códigos de respuesta 
rápida, en inglés quick response code, que a través de una matriz de puntos almacenan una gran cantidad de dígitos y permiten añadir información virtual.

Con base a estos antecedentes, el presente proyecto tiene como objetivo el estudio de factibilidad para publicitar productos empresariales con códigos QR en la ciudad AmbatoEcuador a través de los diferentes estudios, de marco lógico, de mercado, técnico administrativo, económico financiero y la evaluación económica financiera. De ésta forma se resuelve una problemática actual, la insuficiente innovación en los productos publicitarios de las empresas. En la sección desarrollo se encuentra especificada la metodología de búsqueda de información y en los resultados se encuentra la aplicación del marco lógico, estudio de mercado, estudio técnico administrativo, estudio económico, evaluación económica financiera, se brinda posibles herramientas tecnológicas de códigos $Q R$ y, finalmente, las conclusiones del trabajo.

\section{DESARROLLO}

Con ayuda de la herramienta tecnológica Perish (Harzing, 2007), se realizaron búsquedas bibliográficas en Google Académico y Microsoft Academic Search para recopilar información de un conjunto de documentos con mayor número de citas de cada artículo que contienen el objeto de estudio promoción de productos empresariales. Además, se consideraron las recomendaciones planteadas para la precisión del estado del arte propuesto por González Alonso y Pérez González (2014).

A su vez, se aplicó una encuesta estructurada con trece preguntas cerradas de selección simple y múltiple, enfocada a 251 empresas como muestra proyectada a 727 empresas comerciales de electrodomésticos y venta de aparatos electrónicos y celulares de la ciudad de AmbatoTungurahua.

Cabe recalcar que este proyecto se realizó mediante un proceso sistemático, el cual tuvo una etapa inicial el marco lógico con el análisis del problema, involucrados, objetivos, alternativas de las que se seleccionó la más idónea. En el estudio de mercado se estableció el cálculo de la muestra, el análisis e interpretación de la encuesta, para ello se utilizó Excel 2013; conjuntamente al análisis de la demanda, oferta, demanda potencial, precio y comercialización. En el estudio técnico administrativo, se detalló el tamaño y localización óptima, ingeniería del proyecto y la distribución de espacios físicos. En el estudio económico financiero, se determinaron los ingresos brutos, gastos, inversión inicial. Finalmente, en la evaluación económica financiera mediante los evaluadores a tiempo presente y futuro, como se desglosa en el siguiente epígrafe. 


\section{RESULTADOS}

El presente trabajo tuvo como resultados cinco etapas bien definidas las que se resumen en los siguientes puntos:

\subsection{MARCO LÓGICO}

Se realizó una investigación autónoma de fuentes bibliográficas para encontrar a los grupos involucrados en la problemática, siendo los siguientes: empresas por su facilidad de comunicación e información sobre productos y promociones, Gobierno Autónomo Descentralizado Municipal de Ambato (GADMA) para dar a conocer sus servicios y la Policía Nacional para realizar campañas de concientización vial y social, entre otros.

A continuación, se determinó el problema esencial, sus causas y consecuencias, las cuales se plasmaron a través de un árbol de problemas; convirtiéndose en un árbol de objetivos con medios y fines. Posteriormente, se ejecutó un análisis de objetivos que desencadenó en tres alternativas de solución, la más relevante corroboró la factibilidad en la creación de una empresa de asesoramiento en Publicidad y Marketing, utilizando Códigos QR mediante la estructuración de un proyecto socio-productivo en la ciudad de Ambato, en Ecuador.

\subsection{ESTUDIO DE MERCADO}

En esta etapa tuvo lugar la segmentación de mercado, para la cual se tomó de referencia datos del (INEC, 2013) que son los datos actualizados a la fecha de la investigación. Se encontró que en Ecuador durante este año existieron 1.110.934 empresas. Exclusivamente, de la zona 3 conformada por las provincias de Chimborazo, Cotopaxi, Pastaza y Tungurahua, existen 145.124 empresas. Mientras que en la ciudad Ambato, perteneciente a la provincia de Tungurahua, concurren 39.154 empresas, de las cuales 15.782 son empresas comerciales y 706 son de electrodomésticos y venta de aparatos electrónicos y celulares; siendo ésta cifra el mercado objetivo que se proyectó para el año 2015 con 727 empresas, con una muestra de 251 empresas, valor proyectado para el Mercado Objetivo, a las cuales se realizó una encuesta estructurada con 13 preguntas de selección cerrada y múltiple.

De la encuesta se encontró los siguientes resultados:

De 727 empresas que representan el 100\% del mercado objetivo, el 19.05\%, que corresponde a 139 empresas, manifiestan que tienen conocimiento de qué son los Códigos QR, mientras que el $80.95 \%$, que representan a 589 empresas, lo desconoce. Teniendo como referente que la mayoría de empresas desconocen qué son los Códigos QR, quizá no les parece relevante o no conocen las herramientas tecnológicas actuales. Es preciso recalcar que, de las empresas con respuesta afirmativa, requerirían que se les procesen de 21 códigos $Q R$ en adelante a nivel empresarial con un $59.52 \%$ y, de ellas, el $47.62 \%$ que corresponde a 346 empresas están dispuestos a pagar un precio entre 10 a 15 dólares por cada uno debido a su fácil acceso y a una comunicación efectiva en el mercado con un $35.71 \%$ de las empresas. 
También, el $11.90 \%$ de las empresas consideran que las formas tradicionales de publicidad son muy buenas, pero no se sienten completamente satisfechos por lo que se evidencia un nicho de mercado aprovechable. Dentro de los motivos más importantes por los que utilizarían los códigos QR el $85.71 \%$ de las empresas, son la innovación en servicios junto a la facilidad para atraer más clientes y su fidelización. Sin embargo, el 80.95\% equivalente a 589 empresas desconocen qué son los códigos QR, pero están interesados en utilizar nuevas herramientas de publicidad.

Actualmente, los sitios web empresariales son una forma de llevar a cabo el proceso de compra y venta, razón por la cual el $47.62 \%$ que corresponde a 346 empresas desearían que los códigos QR publiciten su empresa por medio de del dominio registrado en internet o URL.

Se encontró la demanda potencial insatisfecha, a partir del cálculo de la demanda y oferta en servicios con los datos de la encuesta, mismos que son restados, como se observa en la tabla 1.

Tabla 1. Demanda Potencial Insatisfecha.

\begin{tabular}{|r|c|c|c|}
\cline { 3 - 4 } AÑO & DEMANDA EN SERVICIOS & $\begin{array}{l}\text { OFERTA EN } \\
\text { SERVICIOS }\end{array}$ & DPI \\
\hline $\mathbf{2 0 1 5}$ & 11.058 & 1.846 & 9.212 \\
\hline $\mathbf{2 0 1 6}$ & 11.218 & 1.873 & 9.346 \\
\hline $\mathbf{2 0 1 7}$ & 11.379 & 1.900 & 9.479 \\
\hline $\mathbf{2 0 1 8}$ & 11.541 & 1.927 & 9.615 \\
\hline $\mathbf{2 0 1 9}$ & 11.706 & 1.954 & 9.752 \\
\hline $\mathbf{2 0 2 0}$ & 11.874 & 1.982 & 9.892 \\
\hline
\end{tabular}

El cálculo de la demanda se realizó en base a las 623 empresas que afirmaron querer adquirir los servicios de asesoría y su frecuencia de consumo, preguntas de la encuesta estructurada. Una vez con ese dato, se procedió al cálculo en base a proyecciones con la tasa de crecimiento empresarial, que se ubica en 1.45\%. Este valor se estableció con base al Directorio de Empresas del año 2013 obtenido del (INEC, 2013) con una simple regla de tres, pues en el año 2012 hubo 696 empresas que se dedicaban al comercio de línea blanca y tecnología; y en el año 2013 existieron 706 empresas que se dedican a la misma actividad. Después, se fusionó con la frecuencia de consumo en la tabla 2, misma que es proyectada también hasta el año 2020.

Tabla 2. Cálculo de la demanda en base a frecuencia de consumo.

\begin{tabular}{|c|c|c|c|c|c|}
\hline \multirow{7}{*}{623} & $\%$ & \multicolumn{2}{|l|}{ FRECUENCIA } & M. F & CONSUMO \\
\hline & 2.38 & 0 a 5 & 14.8 & 2.5 & 37.1 \\
\hline & 14.29 & 6 a 10 & 89.0 & 8 & 712.2 \\
\hline & 4.76 & 11 a 15 & 29.7 & 13 & 385.5 \\
\hline & 19.05 & 16 a 20 & 118.7 & 18 & 2.136 .3 \\
\hline & 59.52 & 21 en adelante & 370.8 & 21 & 7.787 .0 \\
\hline & & & & SUMATORIA & 1.1058 \\
\hline
\end{tabular}

Fuente: elaboración propia. 
Tabla 31. Demanda Proyectada en Servicios.

\begin{tabular}{|c|c|c|}
\hline AÑO & DEMANDA EN SERVICIOS & T.C.E \\
\hline $\mathbf{2 0 1 5}$ & 11.058 & $1.45 \%$ \\
\hline $\mathbf{2 0 1 6}$ & 11.218 & 160.3 \\
\hline $\mathbf{2 0 1 7}$ & 11.379 & 162.7 \\
\hline $\mathbf{2 0 1 8}$ & 11.541 & 165.0 \\
\hline $\mathbf{2 0 1 9}$ & 11.706 & 167.4 \\
\hline $\mathbf{2 0 2 0}$ & 11.874 & 169.7 \\
\hline
\end{tabular}

Fuente: elaboración propia.

En el cálculo de la oferta es similar el procedimiento. Se tomó como referencia las 104 empresas que no desean adquirir los servicios de asesoría y se proyecta también hasta el año 2020.

Tabla 4. Oferta en Servicios.

\begin{tabular}{|c|c|c|c|c|c|}
\hline & $\%$ & FRECUENCIA & & M. F & CONSUMO \\
\hline \multirow{6}{*}{104} & 2.38 & 0 A 5 & 2.48 & 2.5 & 6 \\
\hline & 14.29 & 6 A 10 & 14.86 & 8 & 119 \\
\hline & 4.76 & 11 A 15 & 4.95 & 13 & 64 \\
\hline & 19.05 & 16 A 20 & 19.81 & 18 & 357 \\
\hline & 59.52 & 21 en adelante & 61.90 & 21 & 1.300 \\
\hline & & & & SUMATORIA & 1.846 \\
\hline
\end{tabular}

Fuente: elaboración propia.

Tabla 5. Oferta Proyectada en Servicios.

\begin{tabular}{|c|c|c|}
\hline AÑO & OFERTA EN SERVICIOS & T.C.E \\
\hline 2015 & 1.846 & $1.45 \%$ \\
\hline 2016 & 1.873 & 26.77 \\
\hline 2017 & 1.900 & 27.15 \\
\hline 2018 & 1.927 & 27.54 \\
\hline 2019 & 1.954 & 27.94 \\
\hline 2020 & 1.982 & 28.34 \\
\hline
\end{tabular}

Fuente: elaboración propia.

Para la proyección del precio se utilizaron los porcentajes de inflación por mes del (INEC, 2015), desde noviembre 2014 hasta noviembre 2015, obteniendo una sumatoria de 3.53\%, y el rango de precios que están dispuestas a pagar las empresas encuestadas. Es pertinente indicar que éste rango está entre $\$ 26$ hasta $\$ 30$, por lo que se aplicó un promedio y como resultado el precio para el año 2015 es de \$28, proyectado hasta el 2020.

Tabla 62. Proyección del Precio hasta 2020.

\begin{tabular}{|c|c|c|}
\hline AÑO & PRECIO & INFLACIÓN \\
\hline $\mathbf{2 0 1 5}$ & 28.00 & $3.53 \%$ \\
\hline $\mathbf{2 0 1 6}$ & 28.99 & 0.99 \\
\hline $\mathbf{2 0 1 7}$ & 29.98 & 1.02 \\
\hline $\mathbf{2 0 1 8}$ & 31.00 & 1.06 \\
\hline
\end{tabular}




\begin{tabular}{|l|l|r|}
\hline 2019 & 32.06 & 1.09 \\
\hline 2020 & 33.15 & 1.13 \\
\hline
\end{tabular}

\subsection{ESTUDIO TÉCNICO ADMINISTRATIVO}

En esta fase se determinó el tamaño óptimo del proyecto. De la demanda potencial insatisfecha (DPI), que se obtuvo previamente en el estudio de mercado, solo se toma el $75 \%$ de cada año, pues no se podrá cubrir la demanda en su totalidad en su primer año y siempre debe existir un margen de demanda para extender este nuevo servicio en los próximos años.

Tabla 73. Cálculo DPI Real.

\begin{tabular}{|c|c|c|}
\hline AÑO & DPI & DPI REAL 75\% \\
\hline $\mathbf{2 0 1 5}$ & 9.212 & 6.909 \\
\hline $\mathbf{2 0 1 6}$ & 9.346 & 7.009 \\
\hline $\mathbf{2 0 1 7}$ & 9.479 & 7.109 \\
\hline $\mathbf{2 0 1 8}$ & 9.615 & 7.211 \\
\hline $\mathbf{2 0 1 9}$ & 9.752 & 7.314 \\
\hline
\end{tabular}

Fuente: elaboración propia.

Con el objetivo de determinar la localización de la empresa de asesoramiento en publicidad a través de códigos QR, la cual tendrá como nombre comercial Virtual Code, se realizó una Matriz de Localización Óptima del Proyecto, obteniendo la ubicación más factible ubicada en las calles Bolívar, entre Rocafuerte y Montalvo, en la Ciudad de Ambato, como se muestra a continuación.

Tabla 84. Matriz de Localización Óptima del Proyecto.

\begin{tabular}{|c|c|c|c|c|c|c|c|c|}
\hline $\begin{array}{c}\text { FACTORESO } \\
\text { FUERZAS } \\
\text { DE } \\
\text { RELEVANCLA }\end{array}$ & $\begin{array}{c}\text { PESO } \\
\text { PONDERADO }\end{array}$ & $\begin{array}{c}\text { PESO } \\
\text { LATERAL }\end{array}$ & $\begin{array}{c}\text { BOLIVAR } \\
\text { ENTRE } \\
\text { ROCAFUERTE } \\
\text { Y MONTALVO }\end{array}$ & & $\begin{array}{l}\text { ROCAFUERTE } \\
\text { ENTRE } \\
\text { MONTALVO Y } \\
\text { MARTINEZ }\end{array}$ & & $\begin{array}{l}\text { CEVALLOS } \\
\text { Y JUAN } \\
\text { LEON } \\
\text { MERA }\end{array}$ & \\
\hline 1 & Servicios básicos & 0,25 & 3 & 0,75 & 3 & 0,75 & 3 & 0,75 \\
\hline 2 & $\begin{array}{l}\text { Personal de } \\
\text { Contacto }\end{array}$ & 0,15 & 3 & 0,45 & 3 & 0,45 & 3 & 0,45 \\
\hline 3 & $\begin{array}{l}\text { Disponibilidad de } \\
\text { Arriendos }\end{array}$ & 0,10 & 3 & 0,30 & 2 & 0,20 & 1 & 0,10 \\
\hline 4 & $\begin{array}{l}\text { Precio de } \\
\text { Arriendo }\end{array}$ & 0,05 & 2 & 0,10 & 3 & 0,15 & $\mathbf{1}$ & 0,05 \\
\hline 5 & $\begin{array}{l}\text { Viabilidad del } \\
\text { Local }\end{array}$ & 0,10 & 2 & 0,20 & 1 & 0,10 & 3 & 0,30 \\
\hline 6 & Comunicación & 0,08 & 3 & 0,24 & 3 & 0,24 & 3 & 0,24 \\
\hline 7 & $\begin{array}{l}\text { Permisos de } \\
\text { funcionamiento }\end{array}$ & 0,10 & 3 & 0,30 & 3 & 0,30 & 3 & 0,30 \\
\hline 8 & Impuestos & 0,05 & 3 & 0,15 & 3 & 0,15 & 3 & 0,15 \\
\hline$g$ & Demanda & 0,05 & 2 & 0,10 & 1 & 0,05 & 3 & 0,15 \\
\hline 10 & $\begin{array}{l}\text { Comodidad de } \\
\text { acceso }\end{array}$ & 0,07 & 2 & 0,14 & 2 & 0,14 & 2 & 0,14 \\
\hline$\Sigma$ & TOTAL & 1,00 & 26 & 2,73 & 24 & 2,53 & 25 & 2,63 \\
\hline
\end{tabular}

Fuente: elaboración propia.

La distribución de espacios físicos de la empresa Virtual Code se determinó específicamente de acuerdo al proceso de servicio. Presenta poca mano de obra al tener procesos automatizados a través de los generadores gratuitos existentes en Internet y lectores de 
Códigos QR descargables disponibles en la red. Así mismo, existen tipos de códigos QR que dependen de las necesidades de los clientes, se encuentran: vincular a páginas sociales, videos, textos, páginas web, geolocalización, vcard y demás servicios.

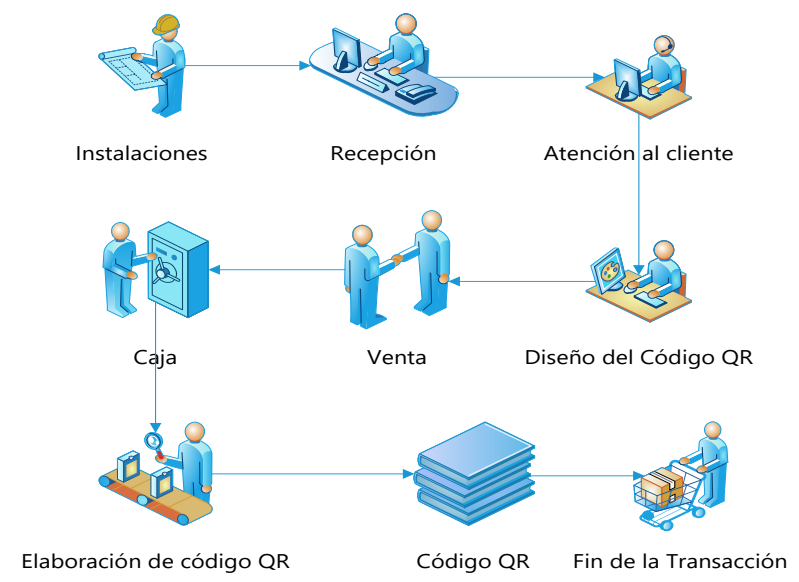

Gráfico 1. Distribución por Servicio.

Fuente: elaboración propia.

Dentro de los requerimientos que la empresa necesita para empezar su funcionamiento se establecieron las necesidades de maquinaria, insumos, equipo de cómputo, muebles y enseres, y mano de obra cualificada, respaldados con sus respectivas proformas para determinar los lugares en los que se adquirirá y la cantidad en unidades físicas. De forma similar, los diagramas de flujo y diagramas de bloques de cada uno de los procesos para la creación de los diferentes tipos de códigos QR.

\subsection{ESTUDIO DE ECONÓMICO}

Se ejecutó el establecimiento de los ingresos brutos desde el año 2015 al 2020 en base a la DPI Real por el Precio.

Tabla 9. Ingresos Brutos.

\begin{tabular}{|c|c|c|c|}
\hline AÑO & DPI REAL & PRECIO & INGRESOS BRUTOS \\
\hline $\mathbf{2 0 1 5}$ & 6.909 & 28.00 & 193.453 .98 \\
\hline $\mathbf{2 0 1 6}$ & 7.009 & 28.99 & 203.185 .05 \\
\hline $\mathbf{2 0 1 7}$ & 7.109 & 29.98 & 213.114 .09 \\
\hline $\mathbf{2 0 1 8}$ & 7.211 & 31.00 & 223.537 .56 \\
\hline $\mathbf{2 0 1 9}$ & 7.314 & 32.06 & 234.470 .53 \\
\hline $\mathbf{2 0 2 0}$ & 7.419 & 33.15 & 245.938 .24 \\
\hline
\end{tabular}

Fuente: elaboración propia.

También se determinaron las unidades monetarias de los gastos de operación, administrativos y financieros, previamente analizados en el estudio técnico administrativo.

Se calculó la inversión inicial de la siguiente forma: 


I. $0=$ ACTIVOS TANGIBLES + ACTIVOS INTANGIBLES + CAPITAL DE TRABANO
I. $0=\$ 18494.53+\$ 5630.00+\$ 4476.58$
I. $0=\$ 28601.11$

Figura 1. Inversión Inicial.

Fuente: elaboración propia.

El financiamiento se planificó en la Cooperativa de Ahorro y Crédito San Francisco, con un Capital de \$28.602 (cifra redondeada para la simulación en línea) a 5 años y con un interés anual del $10.21 \%$. Se precisa que en el proyecto coincidan los valores del Tmar1 y Tmar2, puesto que la empresa sólo tiene financiamiento de la Cooperativa San Francisco y se toma como referencia la tasa activa de la misma.

Todos estos datos se reflejan en el Balance General y generaron valores totales, de activos $\$ 26.812 .55$, pasivos $\$ 31.586 .39$, total pasivos y patrimonio $\$ 26.812 .55$. A su vez, se reflejaron en el Estado de Resultados, donde se obtuvieron flujos netos de efectivo de $\$ 102.023 .42$ para el año 2016; \$107.880.09 para el año 2017; \$114.021.09 para el año 2018; \$120.454.90 para el año 2019 y \$127.213.72 para el año 2020.

\subsection{EVALUACIÓN ECONÓMICA FINANCIERA}

Finalmente, en ésta etapa se elaboró los evaluadores en tiempo presente: índices de solvencia, liquidez, endeudamiento y apalancamiento. Y además evaluadores en tiempo futuro: VAN, TIR, R C/B y PRI.

Dentro de los evaluadores en tiempo presente tenemos:

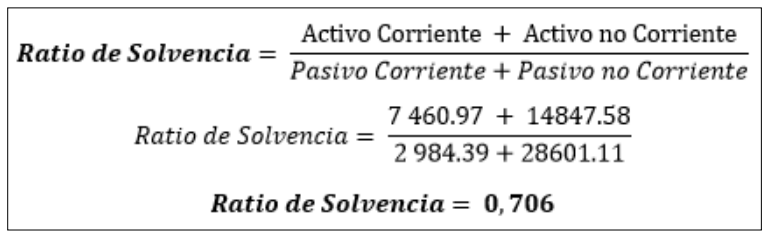

Figura 2. Ratio de Solvencia.

Fuente: elaboración propia.

Este valor evidencia que los pasivos son mayores que los activos, pues la empresa tiene un ratio de solvencia de 0.706 , siendo un valor bajo, pues lo ideal es que sea superior a 1.5. Por lo que la empresa Virtual Code no posee la solvencia necesaria para hacer frente a sus pagos a corto plazo.

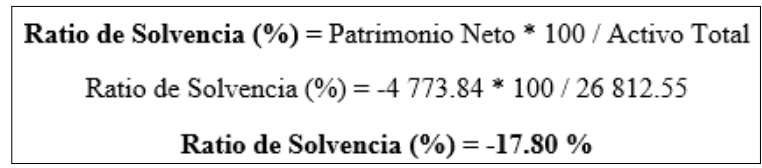

Figura 3. Ratio de Solvencia \%. Fuente: elaboración propia.

La empresa Virtual Code tiene un ratio de solvencia de $-17.80 \%$, valor que es negativo, ya que su patrimonio también es negativo. 


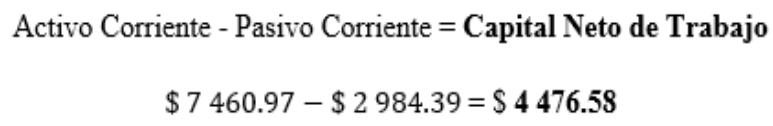

Figura 4. Capital Neto de Trabajo.

Fuente: elaboración propia.

El capital neto de trabajo en la empresa Virtual Code es de $\$ 4.476 .58$, correspondiente al capital con el cual la empresa logrará el normal funcionamiento de sus actividades a corto plazo.

Activo Corriente $/$ Pasivo Corriente $=$ Índice de Liquidez Corriente
$\$ 7460.97 / \$ 2984.39=\$ 2.50$

Figura 5. Índice de Liquidez Corriente.

Fuente: elaboración propia.

El proyecto cuenta con un índice de liquidez corriente de $\$ 2.50$, lo que significa que, por cada dólar de pasivos corrientes tenemos $\$ 2.50$ de activos corrientes para cubrir las obligaciones.

$$
\begin{gathered}
\text { Nivel } \text {. de endeudamiento }=\frac{\text { Total Pasivo }}{\text { Total Activo }} \times 100 \\
\text { Nivel de endeudamiento }=31586.39 / 26812.55 * 100 \\
\text { Nivel de endeudamiento }=117.80 \%
\end{gathered}
$$

Figura 6. Endeudamiento sobre Activos Totales.

Fuente: elaboración propia.

La participación de los acreedores es del 117.80 \% sobre el total de los activos de la empresa; lo cual representa un nivel de riesgo muy alto.

Nivel. de Endeudamiento $=\frac{\text { Total Pasivo }}{\text { Total Patrimonio }}$
Nivel de Endeudamiento $=31586.39 /-4773.84$
Nivel de Endeudamiento $=-\mathbf{6 . 6 2}$ veces

Figura 7. Endeudamiento sobre Activos Totales.

Fuente: elaboración propia.

De los resultados anteriores se concluye que en la empresa para el 2016 su patrimonio supera en 6.62 veces a sus pasivos. Por lo que se observa un gran nivel de endeudamiento en relación a su patrimonio.

$$
\begin{gathered}
\text { Tasa de Deuda }=\frac{\text { Deuda Total }}{\text { Activo Total }} * 100 \\
\text { Tasa de Deuda }=\frac{31586.39}{26812.55} * 100 \\
\text { Tasa de Deuda }=117.80 \%
\end{gathered}
$$

Figura 8. Endeudamiento sobre Activos Totales.

Fuente: elaboración propia. 
El total de fondos provenientes de instituciones de crédito representa un porcentaje elevado del activo total de la empresa; por lo que es un valor alto tomando en cuenta la tasa aceptable. Con lo cual, las instituciones financieras podrían negar el acceso a dar préstamos a una empresa muy endeudada por el riesgo que corren de no recuperar su dinero.

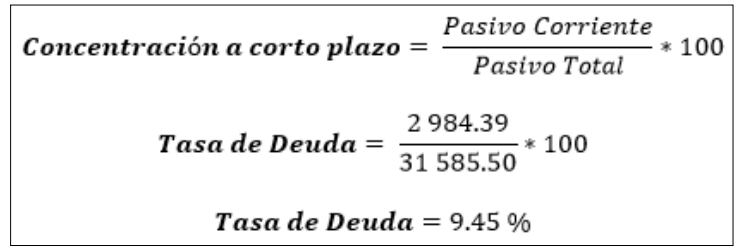

Figura 9. Endeudamiento sobre Activos Totales. Fuente: elaboración propia.

El $9.45 \%$ del total de pasivos de la empresa son corrientes, es decir, que son deudas y obligaciones que mantiene la empresa al corto plazo con terceras personas. Se determina que el endeudamiento de la empresa se concentra en el pasivo fijo, largo plazo con un $9.45 \%$ del total de pasivos.

Dentro de los evaluadores de tiempo futuro se encuentran:

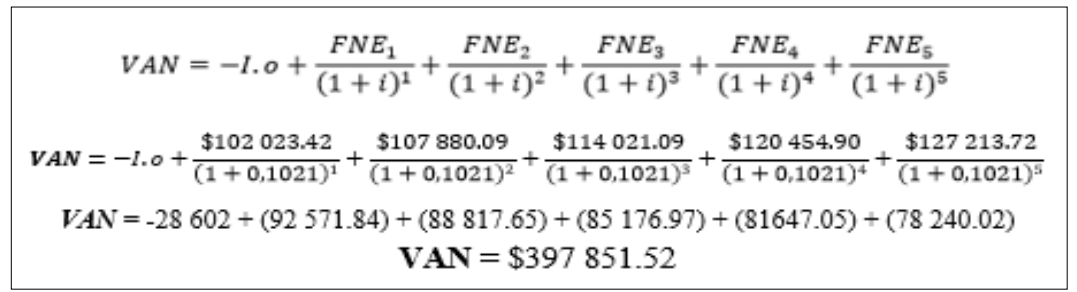

Figura 10. Valor Actual Neto (VAN).

Fuente: elaboración propia.

Tras traer a tiempo presente los flujos de efectivo y descontando la inversión Inicial, se obtiene un rendimiento de $\$ 397,851.52$ dólares en la vida útil del proyecto. Aplicando la Regla de Decisión: VAN $\geq 0$.

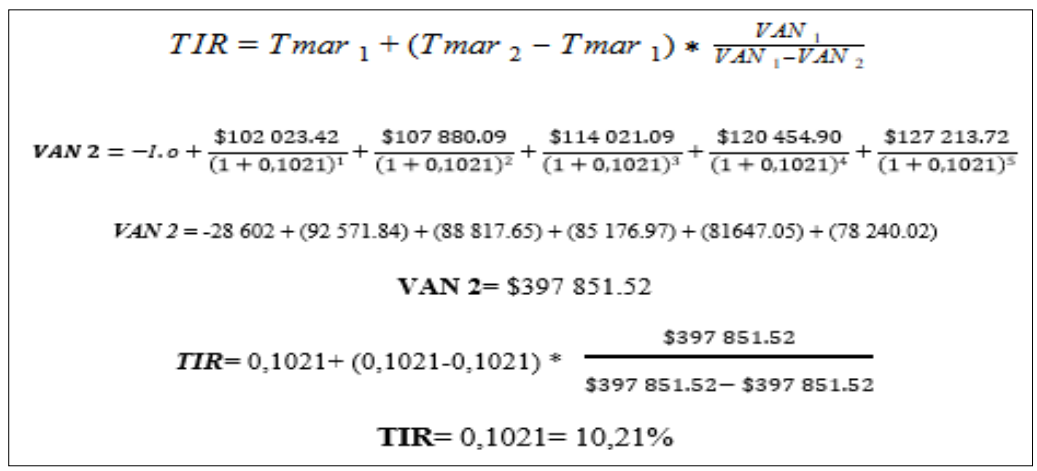

Figura 11. Tasa Interna de Retorno (TIR). Fuente: elaboración propia.

La empresa Virtual Code tendrá una tasa de rendimiento del $10.21 \%$ en los 5 años de vida útil del proyecto. Aplicando la Regla de Decisión: TIR = Tmar. 


$$
\begin{gathered}
\mathbf{R C} / \mathbf{B}=\frac{\Sigma \text { Costos totales }}{\sum \text { ingresos brutos }} \\
\mathbf{R C / B}=\frac{\$ 216605.17}{1313699.45} \\
\frac{\mathbf{R C}}{\mathbf{B}}=\$ 0.17
\end{gathered}
$$

Figura 12. Relación Costo/Beneficio. Fuente: elaboración propia.

Por cada dólar del costo del proyecto obtenemos 0.17 centavos de beneficios. Por lo tanto, se ve la relación de proporcionalidad baja. Aplicando la Regla de Decisión: RC/B $\geq 0$.

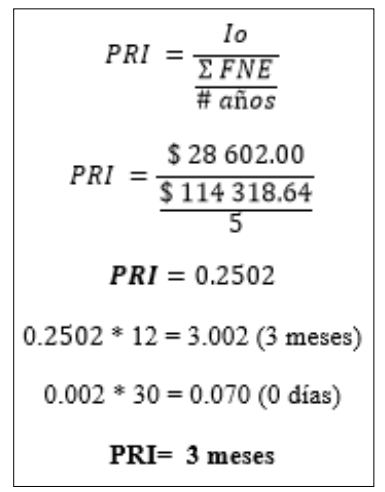

Figura 13. Periodo Promedio de Recuperación.

Fuente: elaboración propia.

Una vez realizado el cálculo se puede evidenciar que la recuperación de la inversión, será de 3 meses, por lo que se recuperará en un periodo de tiempo corto. Aplicando la Regla de Decisión: $\mathrm{PIR} \leq$ Vida útil.

La empresa Virtual Code tiene los siguientes escenarios: pesimista (decremento del 20\%), optimista (incremento 20\%) y el escenario real (estado de resultados 2016). Para los cálculos se tomó los valores del estado de resultados.

\begin{tabular}{|l|l|l|l|}
\hline & PESIMISTA & OPTIMISTA & \multicolumn{1}{c|}{ REALISTA } \\
\hline AÑO 2016 & $\$ 203.185 .05$ & $\$ 243.822 .05$ & $\$ 162.548 .04$ \\
\hline AÑO 2017 & $\$ 213.114 .09$ & $\$ 255.736 .91$ & $\$ 170.491 .27$ \\
\hline AÑO 2018 & $\$ 223.537 .56$ & $\$ 268.245 .07$ & $\$ 178.830 .05$ \\
\hline AÑO 2019 & $\$ 234.470 .53$ & $\$ 281.364 .64$ & $\$ 187.576 .43$ \\
\hline AÑO 2020 & $\$ 245.938 .24$ & $\$ 295.125 .88$ & $\$ 196.750 .59$ \\
\hline
\end{tabular}

Tabla 10. Escenarios.

Fuente: elaboración propia.

\subsection{RECOMENDACIONES EN HERRAMIENTAS TECNOLÓGICAS}

Los códigos QR son medios que ayudan a informar por su alta capacidad de almacenamiento en cuanto a su contenido. Se pueden colocar en paneles, edificios, mapas, folletos, revistas entre otros, con el propósito de difundir información, como lo detalla Beltrán López (2014, pp. 99-100). Por lo tanto, en publicidad y marketing son infinitos los usos de los códigos QR solo 
se necesita creatividad e imaginación. Por ejemplo, se pueden promocionar los productos de las empresas e incluso promover la venta cruzada, de esta manera fidelizar a los clientes.

La estructura básica de un código QR consta de la información de versión, formato, los puntos de datos y errores, la posición, alineamiento, sincronización y la zona de silencio, como se observa en el gráfico 2 .

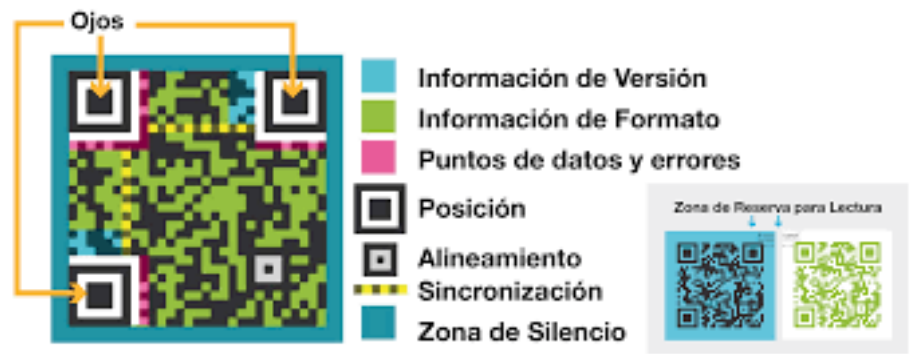

Gráfico 2. Estructura del Código QR.

Fuente: Código QR-Código de Respuesta Rápida, (Grupo OP Gráficas S.A., Junio- 13).

\subsection{GENERADORES DE CÓDIGOS QR}

Se puede crear códigos QR con ayuda de un generador de códigos QR, como manifiestan Carrodeguas (2012); Denso Wave Incorporated (2014). En primer lugar, se deberá seleccionar la función que el código deberá cumplir. En un segundo paso, deberá introducir el contenido que se tenga que codificar antes de poder realizar un diseño individual con los colores y el logotipo que correspondan. Por último, el código se tiene que descargar, momento en el que ya estará listo para imprimirlo. Existen aplicaciones gratuitas y otras muy sofisticadas para generar los códigos QR, siendo importante definir la utilización que se le dará al código QR. Por ejemplo: http://www.qrstuff.com/, http://www.qrcode-monkey.com/es y http://arcode.kaywa.com/, entre otros sitios web.

\subsection{LECTORES DE CÓDIGOS QR}

El importante aporte de Bonet (2010) indica que para leer o interpretar un código QR es necesario un dispositivo con cámara de fotos y un lector compatible. Actualmente, en Internet se pueden encontrar decenas de lectores capaces de leer e interpretar estos códigos. Todo depende del sistema operativo del teléfono móvil y poco más. 
Tabla 11. Lectores de código QR.

\begin{tabular}{|c|c|c|c|}
\hline $\begin{array}{c}\text { Para Android: Barcode } \\
\text { Scanner }\end{array}$ & Para iPhone: i-nigma & $\begin{array}{c}\text { Para Nokia } \\
\text { Symbian: Barcode } \\
\text { Reader }\end{array}$ & $\begin{array}{c}\text { Para } \\
\text { BlackBerry: Beetagg }\end{array}$ \\
\hline $\begin{array}{l}\text { Lector de códigos QR } \\
\text { polivalente. Lee e } \\
\text { interpreta este } \\
\text { estándar, el programa } \\
\text { es capaz de capturar } \\
\text { otros formatos de } \\
\text { códigos de barra y } \\
\text { lanzar búsquedas de } \\
\text { referencias en Internet. }\end{array}$ & $\begin{array}{l}\text { Soporta otros códigos } \\
\text { de barra como } \\
\text { DataMatrix. Programa } \\
\text { ideal para compartir } \\
\text { códigos con otros } \\
\text { usuarios. }\end{array}$ & $\begin{array}{l}\text { Lee códigos QR, BIDI } \\
\text { o DataMatrix. } \\
\text { Guardar los datos de } \\
\text { los códigos QR en } \\
\text { una librería, enviar } \\
\text { códigos por SMS, } \\
\text { correo o Bluetooth. }\end{array}$ & $\begin{array}{l}\text { Lee e interpreta } \\
\text { códigos QR utilizando } \\
\text { la cámara disponible } \\
\text { por defecto en el } \\
\text { dispositivo. } \\
\text { compatible con otros } \\
\text { formatos como } \\
\text { DataMatrix } \\
\text { BeeTagg Code. }\end{array}$ \\
\hline $\begin{array}{l}\text { https://play.google.com } \\
\text { /store/apps/details?id= } \\
\text { com.google.zxing.client. } \\
\text { android\&hl=es }\end{array}$ & $\begin{array}{l}\text { http://i- } \\
\text { nigma.en.softonic.com } \\
\text { Liphone }\end{array}$ & $\begin{array}{l}\text { http://barcode- } \\
\text { reader- } \\
\text { s605th.en.softonic.c } \\
\text { om/symbian }\end{array}$ & $\begin{array}{l}\text { http://www.beetagg } \\
\text { com/en/download- } \\
\text { gr-reader/ } \\
\text { http://get.beetagg.c } \\
\underline{\text { om (Directo desde }} \\
\text { móvil) }\end{array}$ \\
\hline
\end{tabular}

Fuente: elaboración propia.

Tabla 12. Ejemplos de códigos QR.

\begin{tabular}{|c|c|c|c|c|c|}
\hline $\begin{array}{c}\text { URL: } \\
\text { página } \\
\text { web UTA }\end{array}$ & $\begin{array}{l}\text { Perfil social: } \\
\text { Facebook } \\
\text { UTA }\end{array}$ & $\begin{array}{l}\text { Perfil Social: } \\
\text { Twitter } \\
\text { UTA }\end{array}$ & $\begin{array}{c}\text { Posición geográfica: } \\
\text { google maps } \\
\text { UTA }\end{array}$ & $\begin{array}{l}\text { Leer un Texto: } \\
\text { Misión } \\
\text { UTA }\end{array}$ & $\begin{array}{l}\text { Video: } \\
\text { Video } \\
\text { UTA }\end{array}$ \\
\hline 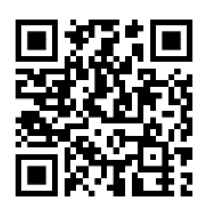 & 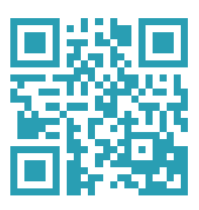 & 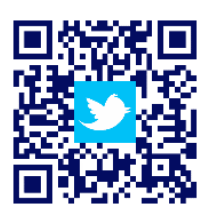 & 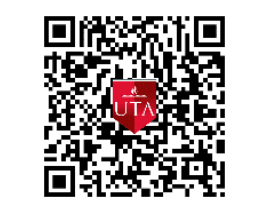 & 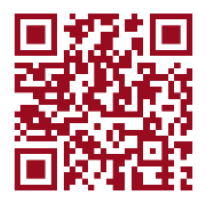 & 口itr \\
\hline
\end{tabular}

Fuente: elaboración propia. 


\section{CONCLUSIONES}

Ecuador constituye un mercado potencial para empresas que publiciten productos empresariales o servicios utilizando estrategias basadas en TIC, el $40.4 \%$ de la población ecuatoriana utiliza internet para obtener información de la web, realizar compras en línea y para comunicarse a través de redes sociales como el Facebook y Twitter (Moya, Escobar, \& Remache, 2015).

Con base a los resultados obtenidos, por medio del marco lógico y los estudios: de mercado, técnico administrativo y económico financiero, sí es factible la creación de una empresa que publicite productos empresariales a través de códigos QR, pues permite satisfacer las necesidades de las empresas con el uso de nuevas herramientas tecnológicas e identifica los productos en el mercado.

A través de la evaluación económica y financiera se determina que el valor actual neto es mayor o igual a cero. En este sentido, la tasa interna de retorno de la inversión es igual a la tasa de descuento que se ofrece a los posibles inversionistas, como se detalla en el estudio económico mediante la TMAR (Tasa Mínima Aceptable de Rendimiento) y la relación costo/beneficio es menor a cero. Finalmente, el período de recuperación de la inversión es de tres meses, por cuanto la inversión inicial es relativamente baja para la rentabilidad que genera el negocio. 


\section{REFERENCIAS BIBLIOGRÁFICAS}

Aching Samatelo, C. (2006). Aplicaciones Financieras de Excel con Matemáticas Financieras. Coordinacion General.

Ávila Macedo, J. J. (2007). Introducción a la Contabilidad. Mexico: Umbral.

Baca Urbina, G. (2010). Evaluación de Proyectos (Sexta). México, DF: McGraw Hill.

Banco Interamericano de Desarrollo. (2001). Estudio de Prefactibilidad Técnica-Económica del Proyecto Fábrica de concentrados para aves de postura. Salvador: Organismo Consultor.

Beltrán López, G. (2014). Geomarketing: geolocalización, redes sociales y turismo. Madrid, España: Creative Commons .

Bonta, P., \& Farber, M. (2002). 199 Preguntas sobre marketing. Madrid: Norma.

Cubbage, F., Financiera, E., \& Frey, G. (2011). Guía para la evaluación económica y financiera de proyectos forestales comunitarios en México. Banco Mundial.

De la Torre, J. (2010). Evaluación de proyectos de inversión. Mexico: Pearson Educación.

Etienne, T. (2010). Financiamiento y Administración de proyectos de Desarrollo (7a ed.). Colombia: Editores Ltda.

Harzing. (2007). Perish. Recuperado 8 de marzo de 2016, a partir de: <http://www.harzing.com/resources/publish-or-perish/windows/>.

Herrera, F., Velasco, C., Denen, H., \& Radulovich, R. (1994). Fundamentos de análisis económico: guía para investigación y extensión rural. Costa Rica: CATIE.

Horne, J., \& Wachowicz, J. (2002). Fundamentos de administración financiera. Mexico: Prentice Hall Inc.

Horngren, Harrison, \& Bamber. (2003). Contabilidad. Mexico: Pearson.

INEC. (2013). Redatam: ECLAC/CELADE. Recuperado 28 de noviembre de 2015, a partir de $<$ http://redatam.inec.gob.ec/cgibin/RpWebEngine.exe/PortalAction?\&MODE=MAIN\&B $\underline{\text { ASE}}=$ DIEE2013\&MAIN=WebServerMain.inl/>.

INEC. (2015). Instituto Nacional de Estadística y Censos. Recuperado 6 de diciembre de 2015, a partir de < http://www.ecuadorencifras.gob.ec/>

Iñigo Carrión y Losune Berastegui. (2010). Guia para la eaboracion de proyectos. México . 
Limas, J. (2012). Marketing Empresarial: Dirección como estratégia Competitiva. Bogotá: Ediciones de la $\mathrm{U}$.

Macchia, J. L. (2005). Cómputos, Costos y Presupuestos. Buenos Aires: Nobuko.

Madurga Bonet, A. (2002). Métodos casuísticos de evaluación de impacto para negociaciones comerciales internacionales. España: INTAL.

Martínez , G., \& Pellicer, E. (2011). Organización y Gestión de proyectos y obras. Madrid: Mc Graw Hill/Interamericana.

Martínez Montes , G., \& Pellicer Armiñana, E. (2011). Organización y Gestión de Proyectos y Obras. Madrid: Mc Graw- Hill.

Merton, R. (2000). Finanzas. Mexico: Pearson.

Moya, E. J. G., Escobar, A. G. A., \& Remache, K. J. O. (2015). Caracterización del consumidor digital ecuatoriano. Revista Publicando, 2(5), 226-242.

Sepulveda, C. (2004). Diccionario de Terminos Economicos. Chile: Universitaria.

Serie de reuniones cursos y conferencias. (1974). Curso Sobre Preparacion y Evaluacion de Proyectos Agropecuarios. Bogota: CIRA.

Sullivan, W., Wicks, E., \& Luxhoj, J. (2004). Ingeniería económica de DeGarmo. México: Pearson.

Zilvetty Torrico , M. (2013). Guía para elaborar proyectos socioproductivos (2a ed.). Bolivia: Educa. 